\title{
How ageing changes the mnemonic bias of visual behaviour
}

\author{
Florence Wedmore
}

Sofía Musil

Division of Brain Sciences, Imperial College London, Charing Cross Campus, St Dunstan’s Rd., London, W6 8RP, UK

\section{David Soto *}

Basque Center on Cognition, Brain and Language, San Sebastian, Spain

Ikerbasque, Basque Foundation for Science, Bilbao, Spain

* Corresponding author:

David Soto, 69 Paseo Mikeletegi, Basque Center on Cognition, Brain and Language, 20009,

San Sebastian, Spain.E-mail: d.soto@bcbl.eu

Email addresses.

florencewedmore@gmail.com, sofia.musil@gmail.com, d.soto@bcbl.eu

RUNNING HEAD: Ageing and mnemonic biases 


\begin{abstract}
Ageing is associated with deficits in cognitive control, including attention and working memory processes. However, how ageing influences the interactions between these cognitive systems is not well understood. The present study compared the oculomotor behavior and search performance of two different age groups in a well established memory-guided visual selection paradigm. The results show that ageing can lead to impairments in the way memory representations bias the control of attention, which notably dissociate in the early stages of oculomotor orientation in search and the later process of attentional disengagement from memory distracters. The implications of these findings for theories of cognitive ageing are discussed.
\end{abstract}




\section{Introduction}

Attention and working memory (WM) functions are fundamental for adaptive behavior. Attention enables the selection of the critical information to meet our goals and the filtering of distracters, while WM allows us to maintain and manipulate the most relevant information 'online' to guide behavior. It is well established that ageing leads to impairments in mechanisms of attentional control, (for a review see (Zanto \& Gazzaley, 2014). One of the key functions of executive control is the filtering of distractors. This filtering process is dependent on individual WM capacity (Vogel \& Machizawa, 2004). The processing capacity of WM declines with increasing age (Hasher, Lynn, \& Zacks, 1988; Salthouse \& Babcock, 1991). Accordingly, a specific impairment in the ability to inhibit and filter out distractors in older adults has been linked to reduced WM capacity (Gazzaley, Cooney, Rissman, \& D’Esposito, 2005; McNab et al., 2015).

The previous research on cognitive ageing examined executive control, WM and attention processes in terms of capacity limitations imposed by age, namely, to prevent distracters from intruding WM interference or the capacity to control the focus of attention within WM representations. But little research to date has explored how cognitive ageing influences the interplay between mnemonic and attentional control systems. A great deal of studies over the last decade have demonstrated that in many circumstances there is an automatic linkage between WM and attention systems in terms of the underlying representations in which they operate (Kiyonaga \& Egner, 2013; Olivers, Peters, Houtkamp, \& Roelfsema, 2011; Soto, Hodsoll, Rotshtein, \& Humphreys, 2008). This body of work illustrated how our attention system can be inadvertently biased to select information that matches the contents held in WM, even when the WM content is known to be detrimental to our search goals. Memory biases of attention affect manual search latencies and the direction of early eye movements made in search, for instance, slowing search responses and directing the eyes away of the search target when a memorymatching distracter is present in the array.

Theories of cognitive ageing are based on impaired inhibitory control (Hasher et al. 2007; Lustig, et al., 2007) or impaired allocation of general cognitive resources or general processing efficiency changes (Salthouse, 1996). Oculomotor control in visual search tasks is one area in which age-related failures of inhibition have been identified. For instance, ageing has been associated with increased oculomotor capture by task-irrelevant salient distracters (Kramer et al. 2000). This notion predicts that WM-biases of attention (i.e. the inadvertent oculomotor orienting towards irrelevant WM contents in the array) should be enhanced with ageing due to a failure to inhibit oculomotor orienting to a stimulus that matches the contents held in memory. Theories of cognitive ageing based on processing efficiency decline (e.g. Salthouse, 1996) also predict that attentional disengagement costs (e.g. once the eyes have been directed to a search distracter matching the contents held in memory) ought to increase with ageing because of 
processing delays to filter out distracting information. This is consistent with findings that distracting stimuli or stimuli that interrupt goal-directed processing impose higher performance costs in ageing than in younger individuals (Clapp, Rubens, Sabarwal, \& Gazzaley, 2011; Clapp $\&$ Gazzaley, 2012). Deficits in distractor disengagement with ageing would also be expected according to Hasher et al. (2007) framework that proposes three critical inhibitory functions compromised in ageing (i) to implement control over prepotent response tendencies (ii) to prevent access of irrelevant items and, crucially, (iii) to delete information that has been attended but that it is no longer relevant.

\section{Methods}

\section{Participants}

11 older ( 7 females) and 11 younger (6 females), healthy volunteers participated in this study. Younger participants were aged between 20 and 31 years (mean 23.9, SDT=4.36). Older participants were aged between 59-81 years (mean 70, SDT=7.22). All participants were right handed and had normal or corrected-to-normal vision. Participants were blinded to the purpose of the study. Participants received informed consent prior to their participation and were financially reimbursed at a rate of 10 pounds per hour.

\section{Apparatus}

The experimental task was run on a PC running Windows 2010. The task was programmed and controlled with E-prime version 2. Eye movements were recorded from both eyes using an infrared eye tracker, at a sampling frequency of $1 \mathrm{kHZ}$ and 12 bits resolution (Jazz Novo, Ober Consulting; spatial resolution: $0.1^{\circ}$ ), which was controlled from a separate computer that was synchronized with the E-prime PC. A chin-rest was used to help participants maintain stable head position during the experimental trials.

\section{Experimental task and procedure}

The task was modelled from a prior study of memory-guided search (Soto, Humphreys, \& Rotshtein, 2007). Each trial began with a fixation display for $500 \mathrm{~ms}$, followed by a cue object which was displayed two times (here 500 and $500 \mathrm{~ms}$, respectively) with a $200 \mathrm{~ms}$ blank interval in between (see Figure 1). This cue could be either valid, invalid or neutral for search performance (see below). After 1 second blank interval, the search display appeared. This contained two black lines presented at the center of each of the two objects, which were placed to the left and right of the fixation point. The object shapes in the search display could be circle, diamond, square, triangle, or hexagon and their colours red, green, blue, yellow, or pink. Objects were displayed on grey background. The distracter lines were vertical, and the sought after target line was tilted $26^{\circ}$ either the left or right. 


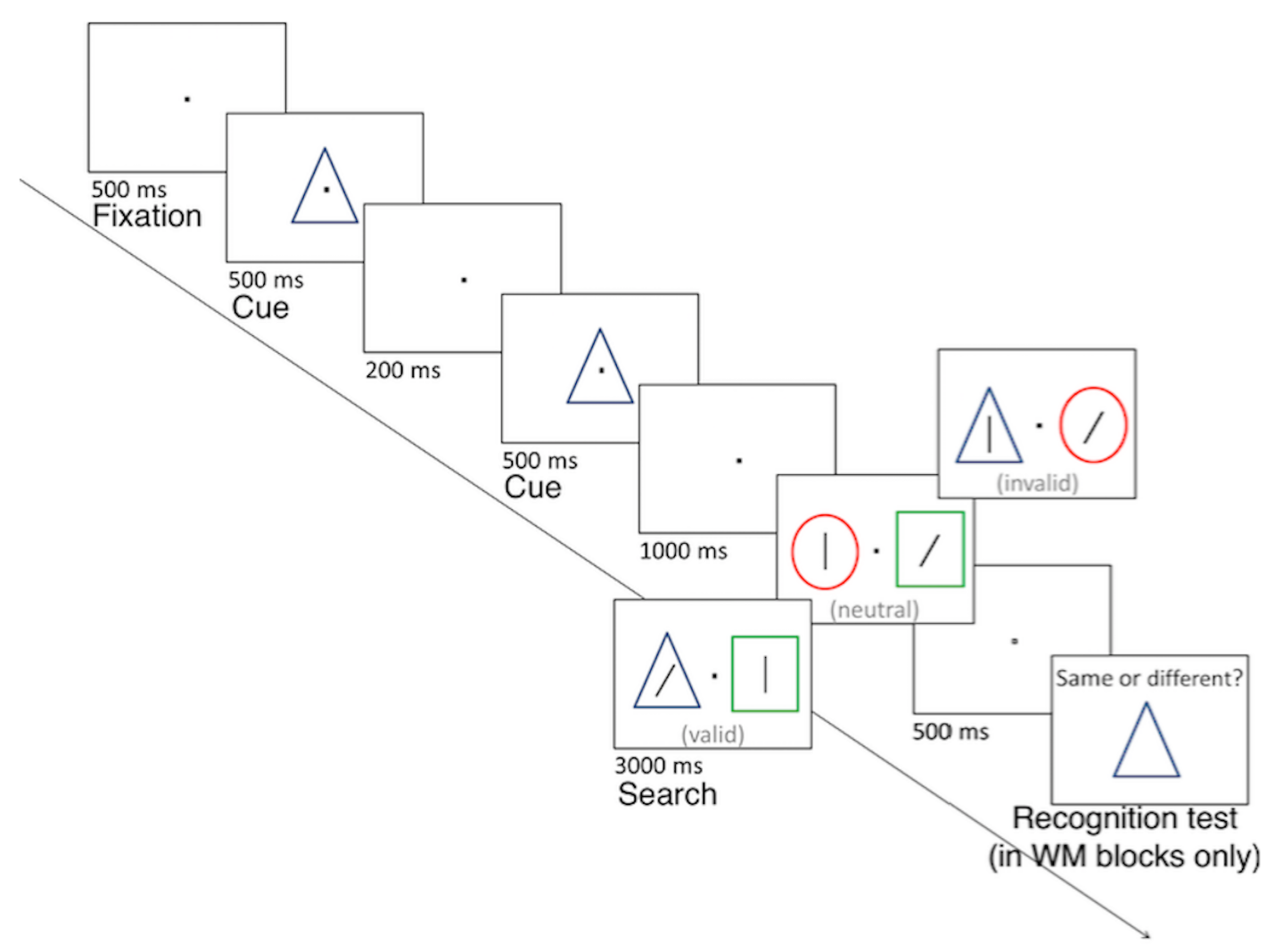

Figure 1. Sequence of events across the different experimental conditions

Each of the objects surrounding the lines was unique in color and shape. Observers had to discriminate the orientation of the target line during a time window of 3 seconds by pressing one of two different buttons, one for left and one for right orientations. The validity of the cue for the search task was varied in three different conditions. In valid trials, the target line appeared within an object that matched both the color and shape of the cue, while during invalid trials the memory cue reappeared in the search display containing a distracter. In the neutral condition, the cue did not reappear in the search display. There was an equal probability of occurrence for the three validity cases.

In the WM condition, observers were required to hold in memory the color and shape of the cue through the trial. In the priming condition, observers were instructed to perceptually compare the two instances of the cue and withhold their response to the search display whenever the second presentation of the cue differed, either in color s1or in shape, from the first presentation. During the WM condition, a memory test followed $500 \mathrm{~ms}$ after completion of the search task. Here, an item was displayed and the observers had to indicate whether it was the same color and shape as the cue or whether it was different (either in color or shape) within an unlimited time window. 
In the priming condition, catch trials $(16.6 \%$ of the total) were introduced to ensure that observers were paying attention to the cues. Catch trials featured two instances of the cue mismatching in either color or shape, and here participants had to withhold their response to the search target. The visual display and events sequence used during the WM and priming conditions was identical for all trials included in the analyses (i.e., the non-catch trials).

The participants completed 6 blocks of WM trials (48 trials per block) and 6 blocks in the priming counterpart (48 trials plus 8 catch trials, per block). In each testing session the two blocks appeared in a randomised order. Each validity condition (invalid, neutral or valid) was weighted to appear equally in each session, in a randomised order. Participants were instructed about the probability of the different validity conditions and were encouraged to perform accurately in all tasks. Speeded responses also were required in the search task.

\section{Saccade detection}

Eye movements were recorded throughout using the Jazz-novo eye tracker with a sampling frequency of $1000 \mathrm{~Hz}$ (Ober Consulting, Poland). The eye tracker was calibrated at the start of each session using the Jazz-recorder software, and re-calibrated if the eye tracker was removed between sessions. Eye movements (saccades) outside of central fixation with a minimum amplitude 1 degree of visual angle were assessed. The parameters used to detect saccades were a minimum fixation of $20 \mathrm{~ms}$, an initial velocity $10 \mathrm{deg} / \mathrm{s}$ and a minimum peak velocity $60 \mathrm{deg} / \mathrm{s}$. Saccades were detected using the saccade detection program in the Jazz-manager software version 3.5 (Ober Consulting, Poland). Eye movement measures were assessed on trials with correct responses in both memory and search tasks. Following Soto et al. (2005) we analysed the direction of the first saccade i.e. towards the target vs towards the distracter as a proxy of early oculomotor orienting. Following Olivers et al. (2006) we assessed the duration of the fixation of the first saccade made to search distracter in order to obtain a marker to attentional disengagement from a search distracter that could or not match the contents held in memory. Also, trials in which no saccade were detected in response to the search display and trials in which the initial saccade latency was over $500 \mathrm{~ms}$ were excluded from eye movement analyses.

\section{Results}

Search accuracy was at ceiling in both the young and older groups (mean proportion correct: 0.99 in both groups).Memory performance in the recognition test was high (mean young: 0.94 and mean old: 0.96 which did not differ, $\mathrm{p}>0.1$ ). Performance in the catch trials in the priming condition was also high (mean old: 0.96 ; mean young: 0.95). These data indicate that both ageing groups followed the instruction of maintaining the cues in the WM condition and withholding responses in the search task during catch trials in the priming condition. Importantly, both groups were matched in these measures of performance. 


\section{Search reaction times $(\mathrm{RTs})$}

A 2 (memory condition: WM, priming) x 3 (cue validity: invalid, neutral and valid) x 2 (age group: young, old) ANOVA was conducted on the median search RTs of the correct responses in both search and memory tests. Figure 2 illustrates the results. Search performance was overall slower in the WM than in the priming condition $\left.\mathrm{F}(1,20)=79.51, \mathrm{p}<.0001, \eta_{\mathrm{p}}{ }^{2}=.79\right)$. This impairment in search performance by WM load was higher in the ageing group, as shown by an interaction between memory condition and the ageing factor $\left(\mathrm{F}(1,20)=10.594, \mathrm{p}<.004, \eta_{\mathrm{p}}{ }^{2}=\right.$. 346). There was an effect of cue validity $\left.\mathrm{F}(2,40)=43.447, \mathrm{p}<.0001, \eta_{\mathrm{p}}{ }^{2}=.685\right)$ with slower performance on invalid relative to the neutral and valid conditions (paired t-tests, all Ps $<$. 0001). Cue validity effects were modulated by the memory condition $(\mathrm{F}(2,40)=21.616, \mathrm{p}=$. $\left.0001, \eta_{\mathrm{p}}^{2}=.519\right)$, indicating that memory biases of search were stronger in the WM relative to the priming condition. There were no further interactions between the above factors and age (lowest $\mathrm{p}$ value $=0.28$ ).
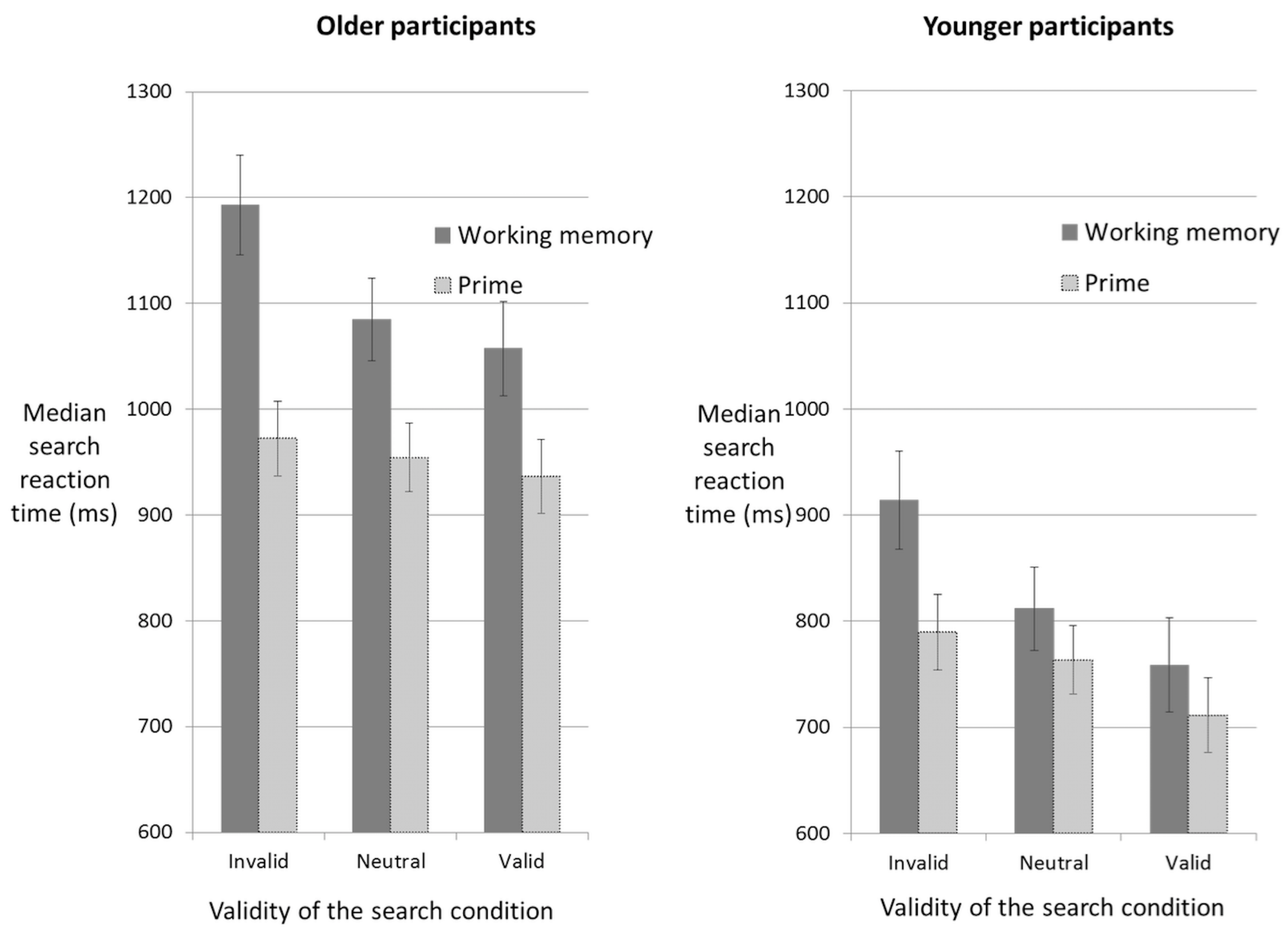

Figure 2. Median search RTs across the validity (invalid, neutral and valid) and task (WM and priming) conditions for each ageing group 


\section{Proportion of first saccades to the target}

The proportion of initial saccades directed to the target were analysed using the same omnibus ANOVA as above in order to find out whether ageing influenced the earliest oculomotor responses driven by memory contents. There was no main effect of memory condition (WM vs priming) on the proportion of first saccades directed towards the target line $\left(F(2,20)=0.409, p=0.53, \eta_{p}^{2}=.02\right)$. There was an effect of validity $\left(F(2,20)=6.07, p<.0001, \eta_{p}{ }^{2}\right.$ $=.75)$; relative to the neutral baseline, the proportion of first saccades to the target was higher on valid trials and lower on invalid trials (paired t-tests, all Ps <.0001). There was an interaction between memory condition and cue validity $\left(\mathrm{F}(2,40)=20.08, \mathrm{p}=.0001, \eta_{\mathrm{p}}{ }^{2}=.5\right)$, showing that the size of the cue validity effect on the proportion of first saccades to the target was highest in the WM case. The cue validity effect was modulated by age $\left(F(2,20)=7.398, p=.006, \eta_{p}^{2}=.27\right)$. Figure 3 depicts the validity effect in WM and priming contexts, across the ageing groups. Notably, however, the effect of validity remained significant in the priming condition only only $\left.(\mathrm{F} 2,40)=19.07, \mathrm{p}<.0001, \eta_{\mathrm{p}}{ }^{2}=.49\right)$ and this was higher in the younger group (i.e. significant interaction between priming validity and age $\mathrm{F}\left(2,40=4.25, \mathrm{p}<0.038, \eta_{\mathrm{p}}{ }^{2}=.175\right)$.

Additional independent sample t-tests showed that both the size of the cue validity effects in the WM and priming conditions (i.e. the difference in the proportion of first saccades to the target in the invalid relative to the valid trials) were reduced in the ageing group relative to the younger group $(\mathrm{t}(20)=2.516, \mathrm{p}=.021$ and $\mathrm{t}(20)=2.282, \mathrm{p}=.034$, two tailed). Further $\mathrm{t}$ tests on the the oculomotor costs (performance invalid - performance neutral) showed a reduction of the WM costs in the ageing group $(t(20)=2.943, \mathrm{p}<.008$. There were no difference in the priming costs across ageing groups ( $>$.25). However the size of the oculomotor benefits (performance neutral - performance valid) in the priming condition was reduced in the ageing group $(\mathrm{t}(20)=2.691, \mathrm{p}<.014)$, while no differences were observed in the WM case ( $\mathrm{p}>.35)$.

The 3-way interaction between memory condition, cue validity and age was non-significant $\left(\mathrm{F}(2,40)=1.293, \mathrm{p}=0.281, \eta_{\mathrm{p}}{ }^{2}=.06\right)$. These results indicate that the early capture of attention by memory-matching items attenuates with age and this effect is similar for representations held in WM and for items that have been merely attended and hence primed. 

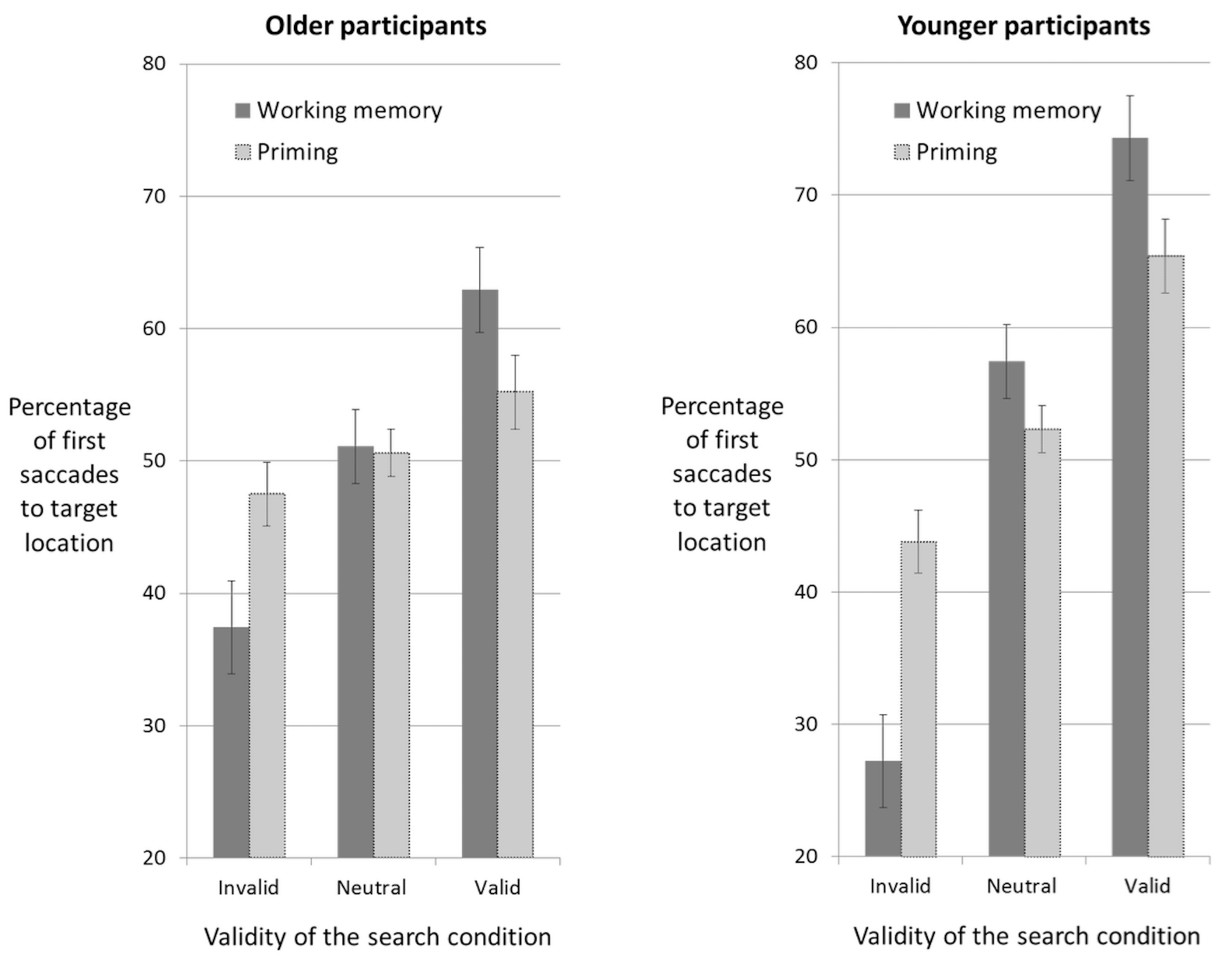

Figure 3. Proportion of first saccades to the target's location across the different experimental conditions and ageing groups.

\section{Fixation durations on a search distracter}

A similar ANOVA was used to assess ageing effects on the duration of fixation of the first saccade made to a search distracter. There was a main effect of memory condition $(F(1,20)=$ $\left.9.639, \mathrm{p}=.006, \eta_{\mathrm{p}}{ }^{2}=.325\right)$ with longer fixation times on a search distracter in the WM relative to the priming condition. There was also an effect of validity $\left(F(1,20)=16.797, p=.001, \eta_{p}{ }^{2}=\right.$. $456)$ with higher duration of fixations in the invalid relative to the neutral $(\mathrm{p}<.0001)$ and the valid condition $(\mathrm{p}<.0001)$ which did not differ $(\mathrm{p}=0.109)$. There was further a memory condition by validity interaction $\left(\mathrm{F}(2,40)=7.83, \mathrm{p}=.001, \eta_{\mathrm{p}}{ }^{2}=.281\right)$ which was also modulated by the age group $\left(\mathrm{F}(4,40)=4.486, \mathrm{p}=.019, \eta_{\mathrm{p}}^{2}=.183\right)$.

To further examine the source of this interaction we computed the costs (invalid fixation duration - neutral fixation durations) and benefits (neutral fixation duration - valid fixation 
duration) across each of the memory conditions and performed independent sample t-tests across the two groups of participants to assess the influence of ageing. The size of the WM cost on fixation duration was bigger in the ageing relative to the younger group $(\mathrm{t}(20)=2.603, \mathrm{p}<.017$, two tailed). However the same effect was not apparent in the priming condition $(t(20)=0.164$, $\mathrm{p}=0.872$ ). Moreover, there were no benefits from valid cueing (neutral fixation duration - valid fixation durations) on the time to disengage attention from a distracter $\mathrm{p}>.93$ and $\mathrm{p}>.55$ in the WM and priming cases respectively.
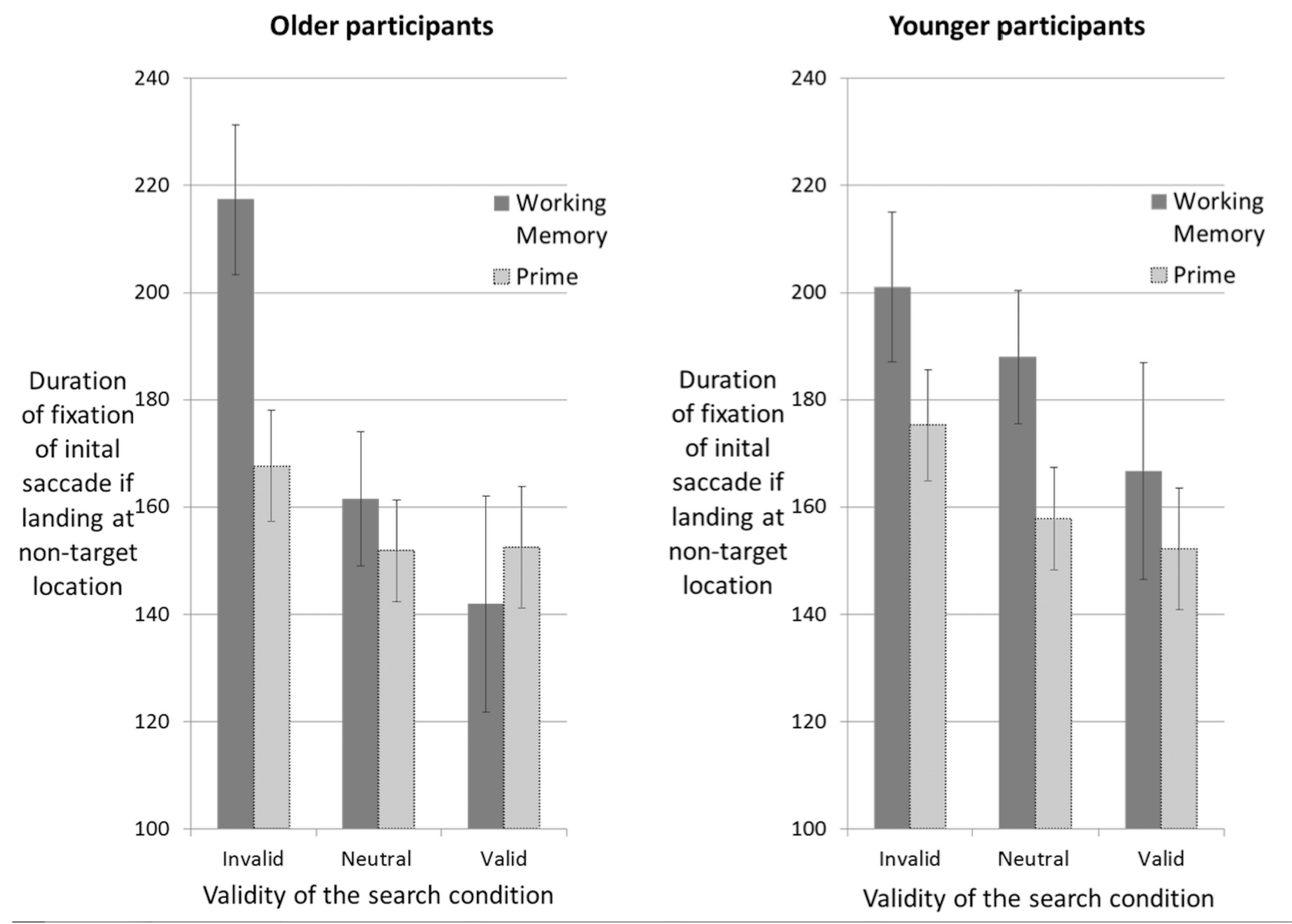

Figure 4. Duration of fixation of initial saccades landing at a non-target location.

\section{Discussion}

Search performance indexed by manual reaction times to identify the search target was strongly modulated by the validity of a match between the memory contents and the visual array. The size of the memory validity effect was stronger when the cue was held in WM relative to the priming condition, in keeping with prior evidence of WM biases of visual selection (Kiyonaga \& Egner, 2013; Olivers, Peters, Houtkamp, \& Roelfsema, 2011; Soto, Hodsoll, Rotshtein, \& Humphreys, 2008). This pattern of search latencies was similar across ageing 
groups, which did not differ also in their memory performance in the recognition test that followed the search task.

WM biases of search latencies attenuate with time (Han \& Kim, 2009). Accordingly, given that the ageing group was overall slower than the younger group, the WM bias of the ageing group observed in the search latencies may have attenuated more with time than in the younger group. Moreover, that the early oculomotor orienting towards a memory matching item was curtailed in the ageing group would have also reduced the overall memory validity effects found in manual search responses. Taken together, these factors could at least partially explain why the observed WM bias in search latencies was not higher in the ageing relative to the younger group

Notably, age-related effects emerged in measures of oculomotor behavior, namely, how memory representations guided the eye movements in the search task. Both age groups demonstrated that the direction of the early saccades in search was biased by the presence of a memory-matching item - leading to a higher proportion of first saccades to the search target on valid trials relative to when invalid cues were held in memory. Previous studies indicated that memory-based effects on eye movements are critically due to the active WM maintenance of the cue not to feature priming (Hollingworth et al., 2013). This is in contrast with the work of Silvis et al. (2015) who did find priming effects on oculomotor biases. Our results also show that priming may be sufficient to trigger a memory bias of oculomotor orienting, however, it is clear from our results that WM-based feedback can enhance this bias. Additional research is needed to pinpoint the factors that lead to the observation of priming effects on oculomotor biasing.

More relevant to the present study is the finding that older participants showed a reduction in this memory bias of early saccades: cue validity effects were lower in the ageing group relative to the younger group. Notably, once the first saccade made in search landed at a non-target location, the older participants fixated the distracter for a longer duration relative to the younger group, particularly when the distracter matched the contents held in WM. This disengagement cost was higher when the distracter was held in WM as opposed to being merely primed.

Taken together, these results suggest that early biases in the orientation of overt attention driven by representations held in memory attenuate with age while the process of disengagement following the capture of attention by a memory distracter also impairs leading to increased disengagement cost. These results are in keeping with the view that ageing impairs how memory representations are used to guide behavior, but critically, they also indicate that the impact of ageing on the processes of attention orienting/capture and disengagement can be dissociated.

Could these results be explained by a reduction in WM capacity with ageing? We believe this is unlikely since the WM load in this study was very low (i.e. a single item) and should not stress the capacity of WM. However it is possible that the fidelity of WM representations was attenuated in the ageing group (Peich, Husain, \& Bays, 2013), which could lead to a reduction in WM guidance. Note however that ageing effects appeared specific to a reduction in memory- 
driven oculomotor capture because the ageing effect was not found in search RTs. Moreover, the disengagement of the eyes from a WM matching item was more costly in the ageing group, which seems difficult to be merely explained by impaired fidelity of a WM representation. This complex pattern of results indicates that impaired WM fidelity is unlikely to be the only factor to account for the reduction of memory biasing of oculomotor behaviour in the ageing group.

These findings are somehow difficult to explain according to existing accounts of cognitive ageing that are primarily based on impaired inhibitory processes (Hasher et al., 2007; Lustig, Hasher \& Zacks, 2007). According to inhibition theory, ageing should be associated with exacerbated oculomotor orienting towards irrelevant WM contents. This was not the case. Several studies indicate that ageing can lead to impaired top-down control and hence increased stimulus-driven attentional capture compared with younger adults (Pratt \& Bellomo, 1999; Iarocci, Enns, Randolph and Burack, 2009; Lincourt, Folk, Hoyer, 1997). Stimulus-driven capture of the eyes by search distracters can also increase in ageing individuals particularly when distracters are more salient (Kramer et al. 2000). But this pattern of increased captured with age with was not supported by the present findings. Here memory-driven biases of early saccades in search either based on repeated exposure (priming) or working-memory contents were lower in the older group compared to the younger group. A key difference between this study and the previous research is the type of paradigm used to manipulate the operation of attention. Here, attention capture was driven top-down by representations held in memory, while in previous studies which reported ageing-related increased in attention and oculomotor capture involved paradigms in which attention was driven bottom-up by a salient stimulus (e.g. Kramer et al. 2000). The inhibition framework of cognitive ageing may need to consider the type of process and content that are to be inhibited in order to optimise performance. The present study shows that information that is irrelevant for our attention goals but that is concurrently held in memory appears to be ignored more easily as we age

The available neuroscientific knowledge on the distinct brain networks supporting stimulusdriven and feature-based memory driven capture of attention can help to explain why and how ageing effects on these forms of attention control may be dissociated. Neural mechanisms of feature-based memory-based biases of visual selection are dissociable from the dorsal and ventral fronto-parietal networks involved in stimulus-driven attentional control (Corbetta and Shulman, 2002). Feature-based mnemonic biasing also involve frontal substrates but, crucially, subcortical substrates in thalamic nuclei can play a fundamental role (de Bourbon-Teles et al., 2016; Soto et al., 2007), including anterior and ventrolateral thalamus involved in memory and oculomotor control (Aggleton et al., 2010; Kunimatsu and Tanaka, 2010), and also the hippocampus (Soto et al. 2012; Stokes et al., 2012). Previous studies of attention control in ageing have mainly focused on deficient deficient frontoparietal control networks involved in attention and the compensatory mechanisms in these frontoparietal networks that may be 
triggered in response to decline in bottom-up processing (see Grady, 2012, Madden, 2007, for reviews). By comparison, research aimed at linking age-related changes in cortico-subcortical connectivity and cognitive function has been scarce, though age-related decreases in processing speed have been related to thalamic and prefrontal volume (Van Der Werf et al., 2001). It is possible that the different brain systems that support both stimulus-driven and feature-based memory driven attention capture are impacted by age in different ways. Accordingly, bottom-up capture may increase with age due to impairments in fronto-parietal attention control networks, while feature-based memory driven capture may attenuate with ageing (e.g.) if ageing leads to attenuation and/or slowing of the feedback processes between a fronto-thalamic network and visual cortex that are critical for memory biasing of attention. This interpretation is also in keeping with the view that ageing impairs the neural manifestation of mental imagery in sensory cortices including a reduction in functional connectivity between prefrontal regions and the relevant visual cortical area for imagery (Kalkstein et al. 2011).

From this perspective, the attenuation of memory-driven saccades with ageing may not be accounted within an inhibition framework but rather be the consequence of age-related decline in feedback excitation driven by memory, perhaps also coupled with an attenuation of the fidelity of the memory representation (Peich, Husain, \& Bays, 2013). This account is also consistent with the view that impaired general processing efficiency mediates cognitive ageing (e.g. Salthouse, 1996). Decline in the structural integrity of white-matter tracts with ageing (Rabbitt et al. 2007; Turken et al., 2008; Van Der Werf et al., 2001) can account for this generalized slowing of brain computations needed for feedback processing and would also explain the ageing-related increase of disengagement costs following capture of the eyes by distracters held in WM(see also Cashdollar et al., 2013). Deficits in disengaging attention with ageing are most likely mediated by impaired frontoparietal networks implicated in selection and executive control, which may fail to suppress distracter-related activity in visual cortex (Gazzaley et al., 2005; Zanto et al., 2011).

One limitation of this study is the relatively small sample of the ageing group which may not fully capture the heterogeneity of the ageing population. Further research using large-scale samples could also adopt an inter-individual differences approach to determine the psychometric structure of memory/attention interactions in relation to cognition more broadly. This could be done by using the combined memory/attention paradigm alongside a comprehensive battery of tasks tapping on different components of memory, attention and executive control, and assessing cross-task performance correlations across different ageing groups. This approach would allow for instance to evaluate how individual measures of attention biasing driven by memory relates to specific components of cognitive control and even to cognition more generally, and, crucially how the psychometric structure supporting memory/attention interactions is impacted by age. 


\section{Acknowledgements}

D.S. acknowledges support from the Spanish Ministry of Economy and Competitiveness, through the 'Severo Ochoa' Programme for Centres/Units of Excellence in R\&D (SEV-2015-490) and project grant PSI2016-76443-P.

\section{References}

Aggleton J.P., O’Mara S.M., Vann S.D., Wright N.F., Tsanov M., Erichsen J.T. (2010) Hippocampal-anterior thalamic pathways for memory: uncovering a network of direct and indirect actions. European Journal of Neuroscience, 31, 2292-2307

Cashdollar, N., Fukuda, K., Bocklage, A., Aurtenetxe, S.K., Vogel, E. \& Gazzaley, A. (2013). Prolonged disengagement from attentional capture in normal aging. Psychology and Aging, 28(1), 77-86.

Clapp, W.C., Rubens, M.T., Sabarwal, J., Gazzaley, A. (2011). Deficit in switching between functional brain networks underlies the impact of multitasking on working memory in older adults. Proceedings of the National Academy of Science USA, 108 (17), 7212-7217.

Clapp, W.C., Gazzaley, A. (2012). Distinct mechanisms for the impact of distraction and interruption on working memory in aging. Neurobiol Aging, 33(1), 134-48.

Corbetta, M., Shulman, G.L. (2002). Control of goal-directed and stimulus-driven attention in the brain. Nature Reviews Neuroscience, 3, 201-215.

de Bourbon-Teles, J., Bentley, P., Koshino, S., Dutta, A., Shah, K., Malhotra, P., Egner, T., Husain, T., Soto, D. (2014). Thalamic control of human attention driven by memory and learning. Current Biology, 4, 993-9.

Gazzaley, A., Cooney, J. W., Rissman, J., D’Esposito, M. (2005). Top-down suppression deficit underlies working memory impairment in normal aging. Nature Neuroscience, 8(10), 1298-1300. 
Grady C. (2012). The cognitive neuroscience of ageing. Nature Reviews Neuroscience, 13(7), 491-505.

Han, S. W., Kim, M.-S. (2009). Do the contents of working memory capture attention? Yes, but cognitive control matters. Journal of Experimental Psychology: Human Perception and Performance. 35(5),1292-1302.

Hasher, L., Lynn, H., Zacks, R. T. (1988). Working Memory, Comprehension, and Aging: A Review and a New View. In Psychology of Learning and Motivation (pp. 193-225).

Hasher , L. , Lustig, C. , Zacks, J. M. (2007). Inhibitory mechanisms and the control of attention. In A. Conway , C. Jarrold , M. Kane , A. Miyake , and J. Towse (eds.), Variation in Working Memory (pp. 227-249 ). New York: Oxford University Press.

Hollingworth, A., Matsukura, M., Luck, S.J. (2013). Visual Working Memory Modulates Rapid Eye Movements to Simple Onset Targets. Psychological Science, 24(5), 790-796.

Iarocci, G., Enns, J. T., Randolph, B., and Burack, J. A. (2009). The modulation of visual orienting reflexes across the lifespan. Developmental Science 12, 715-724.

Kalkstein , J., Checksfield, K., Bollinger, J., Gazzaley, A. (2011). Diminished top-down control underlies a visual imagery deficit in normal aging. Journal of Neuroscience, 31, 15768-15774.

Kiyonaga, A., Egner, T. (2013). Working memory as internal attention: toward an integrative account of internal and external selection processes. Psychonomic Bulletin \& Review, 20(2), $228-242$.

Kramer, A. F., Hahn, S., Irwin, D. E., Theeuwes, J. (2000). Age differences in the control of looking behavior: Do you know where your eyes have been? Psychological Science, 11, 210217.

Kunimatsu J., Tanaka M. (2010) Roles of the primate motor thalamus in the generation of antisaccades. Journal of Neuroscience, 30, 5108-5117

Lincourt, A., Folk, C, Hoyer, J. (1997). Effects of aging on voluntary and involuntary shifts of attention. Aging, Neuropsychology and Cognition, 4, 290-303. 
Lustig, C., Hasher, L., \& Zacks, R. T. (2007). Inhibitory deficit theory: Recent developments in a "new view". In D. S. Gorfein \& C. M. MacLeod (Eds.), The place of inhibition in cognition. Pp. 145-162. Washington, DC: American Psychological Association.

Madden, D.J. (2007) Aging and Visual Attention. Current Directions in Psychological Science, $16,70-74$.

McNab, F., Zeidman, P., Rutledge, R. B., Smittenaar, P., Brown, H. R., Adams, R. A., Dolan, R. J. (2015). Age-related changes in working memory and the ability to ignore distraction. Proceedings of the National Academy of Sciences of the United States of America, 112(20), $6515-6518$.

Olivers, C. N. L., Meijer, F., Theeuwes, J. (2006). Feature-based memory-driven attentional capture: visual working memory content affects visual attention. Journal of Experimental Psychology: Human Perception and Performance, 32(5),1243-65.

Olivers, C. N. L., Peters, J., Houtkamp, R., Roelfsema, P. R. (2011). Different states in visual working memory: when it guides attention and when it does not. Trends in Cognitive Sciences, 15(7), 327-334.

Peich, M-C, Husain, M., Bays, P.M. (2013). Age-Related Decline of Precision and Binding in Visual Working Memory. Psychology and Aging, 28(3), 729-743.

Pratt, J., Bellomo, C. (1999). Attentional capture in younger and older adults. Aging. Neuropsychology and Cognition, 6, 19-31.

Rabbitt, P. , Lunn, M. , Pendleton , N. , Horan, M. , Scott, M. , Thacker , N. , Lowe , C. , Jackson, A. (2007a). White matter lesions account for all age-related declines in speed but not in intelligence. Neuropsychology, 21, 363-370 .

Salthouse, T. A. (1996). Constraints on theories of cognitive aging. Psychonomic Bulletin \& Review, 3(3), 287-299.

Salthouse, T. A., Babcock, R. L. (1991). Decomposing adult age differences in working memory. Developmental Psychology, 27(5), 763-776.

Silvis, J. D., Belopolsky, A. V., Murris, J. W., Donk, M. (2015). The Effects of Feature-Based Priming and Visual Working Memory on Oculomotor Capture. PloS one, 10(11), e0142696. 
Soto, D., Heinke, D., Humphreys, G.W., Blanco, M.J. (2005). Early, involuntary top-down guidance of attention from working memory. Journal of Experimental Psychology: Human Perception and Performance, 31, 248-261.

Soto, D., Humphreys, G.W., Rotshtein, P. (2007). Dissociating the neural mechanisms of memory-based guidance of visual selection. Proceedings of the National Academy of Sciences, USA, 104, 17186-91.

Soto D., Greene, C.M., Kiyonaga, A., Rosenthal, C.R., Egner, T. (2012) A parieto-medial temporal pathway for the strategic control over working memory biases in human visual attention. Journal of Neuroscience, 32, 17563-71.

Soto, D., Hodsoll, J., Rotshtein, P., Humphreys, G.W. (2008). Automatic guidance of attention from working memory. Trends in Cognitive Sciences, 12(9), 342-348.

Soto, D., Humphreys, G.W., Rotshtein, P. (2007). Dissociating the neural mechanisms of memory-based guidance of visual selection. Proceedings of the National Academy of Sciences of the United States of America, 104(43), 17186-17191.

Stokes, M.G., Atherton, K., Patai, E.Z., Nobre, A.C. (2012) Long-term memory prepares neural activity for perception. Proceedings of the National Academy of Sciences of the United States of America, 109, 360-367.

Turken, A. U., Whitfield-Gabrieli , S., Bammer, R., Baldo, J.V., Dronkers, N. F., Gabrieli , J. D. E. (2008). Cognitive processing speed and the structure of white matter pathways: Convergent evidence from normal variation and lesion studies. NeuroImage, 42, 1032-1044 .

Van Der Werf, Y.D., Tisserand, D.J., Visser, P.J., Hofman, P.A., Vuurman, E., Uylings, H.B., Jolles, J. (2001).Thalamic volume predicts performance on tests of cognitive speed and decreases in healthy aging. A magnetic resonance imaging-based volumetric analysis. Cognitive Brain Research, 11(3), 377-85.

Vogel, E. K., \& Machizawa, M. G. (2004). Neural activity predicts individual differences in visual working memory capacity. Nature, 428(6984), 748-751. 
Zanto, T.P. \& Gazzaley, A. (2014). Attention and aging. In: Nobre, A.C. \& Kastner, S. (Eds.). Handbook of Attention, pp. 927-971. Oxford, UK: Oxford University Press.

Zanto, T.P., Rubens, M.T., Thangavel, A., Gazzaley, A. (2011). Causal role of the prefrontal cortex in top-down modulation of visual processing and working memory. Nature Neuroscience, 14(5), 656-661.

Gazzaley, A., Cooney, J.W., Rissman, J., D’esposito, M. (2005). Top-down suppression deficit underlies working memory impairment in normal aging. Nature neuroscience, 8(10), 1298-1300. 